XVII.

Aus der I, chirurgischen Universitäts-Klinik in Wien

(Vorstand: Prof. v. Eiselsberg).

\title{
Ueber die Bedeutung der Milz in dem an malignem Tumor erkrankten Organismus und die Beeinflussung von Tumoren durch Milzbrei.
}

\author{
Von \\ Dr. E. G. Oser und Dr. Egon Ewald Pribram, \\ Operateure der Klinik. \\ (Mit 8 Abbildungen im Text.)
}

Gelegentlich pathologisch-anatomischer Sectionen oder bei operativen Eingriffen wegen maligner Tumoren kann man sich oft überzeugen, dass Carcinome und Sarkome in gewissen Organen äusserst selten, sowohl primär wie metastatisch vorkommen. Eine Erklärung dieser Resistenz könnte vielleicht in der chemischen Beschaffenheit der Zellen oder auch in der Bildung gewisser Antikörper zu suchen sein.

So fand Oestreich bei Sectionen von an Carcinom Verstorbenen, dass auffallenderweise Knorpel und Gefässwände fast stets vom Tumor verschont blieben. Es gelang ihm, aus diesen Organen die Chondroitinschwefelsäure darzustellen, auf deren Vorhandensein er die Resistenzfähigkeit dieser Zellen zurückführt. Oestreich versuchte nun ein Präparat aus der Chondroitinschwefelsäure herzustellen und gab sich der Hoffnung hin, eine Beeinflussung des Carcinoms damit erzielen zu können. Nach seinen Angabęn stellte die Firma Riedl das "Antituman" her, das aus

Natrium chondroidino-sulf. 0,1 ,

$\beta$-Eucain 0,01 ,

Aq. dest. ad 4,0

zusammengesetzt ist. Er soll damit gute Erfolge erzielt haben und empfahl es sowohl zur Nachbehandlung operirter wie auch inoperabler Fälle. Wir hatten Gelegenheit, an der I. chirurgischen Klinik in Wien (Prof. v. Eiselsberg) eine Reihe von inoperablen Fällen von Carcinom und auch einige Recidive damit zu behandeln und möchten im Folgenden kurz über unsere Resultate berichten.

Unsere therapeutischen Versuche erstreckten sich auf 18 Patienten, darunter :

9 Recidive von Carcinoma mammae,

2 Scirrhen der Mamma,

2 Mundboden-Carcinome,

1 Carcinom, das von den Tonsillen ausging.

Genau nach den Angaben von Oestreich wurde das Antituman, entfernt von der erkrankten Stelle, durch etwa 4-8 Wochen intra- 
musculär injicirt. Unsere Resultate waren recht unbefriedigend. In keinem dieser Fälle konnte auch nur ein Stillstand im Wachsthum oder gar eine Rückbildung der Metastasen beobachtet werden. Es muss jedoch erwähnt werden, dass die Patienten etwa eine Stunde nach der Injection über Schmerzen im Tumor klagten und Temperatursteigerungan bis $\mathrm{zu}$ $38^{\circ}$ und Pulsbeschleunigung zeigten.

Wenn auch das Antituman eine Beeinflussung des Carcinoms nicht herbeiführen kann, so ist damit keineswegs ausgeschlossen, dass die Resistenz, die Knorpel und Gelässwände dem Uebergreifen des Tumors entgegensetzen, ausschliesslich in der chemischen Beschaffenheit der Zellen bezw. im Gehalt an Chondroitinschwefelsäure seinen Grund hat.

Auch die Milz wird nur selten von malignen Tumoren ergriffen. Derselben kommt auch sonst insofern eine Sonderstellung im Körper zu, als sie eine hervorragende Rolle im Kampfe des Organismus gegen Toxine verschiedenster Art spielt.

Die Milz ist bekanntlich nebst dem Knochenmark als Hauptbildungsstätte von Antikörpern anzusehen, und man kann sich auch leicht experimentell daron überzeugen, dass z. B. die Agglutinine in der Milz viel früher als im Blute nachzuweisen sind. Es wäre daher auch denkbar, dass in einem an Carcinom oder Sarkom erkrankten Organismus gewisse Substanzen in der Milz gebildet werden, denen eine ähnliche Rolle wie den Antikörpern zukommt. Die Bildung derartiger Substanzen könnte dann die erhöhte Resistenzlähigkeit dieses Organs gegenüber malignen Tumoren erklären.

Von diesem Gedanken ausgehend, hat Braunstein es unternommen, der Frage auf experimentellem Wege näher zu treten und zu erproben, ob durch Milzbrei eine Beeinflussung von malignen Tumoren zu erzielen sei. Derartige Versuche liessen sich naturgemäss nur am Thiere ausführen und wenn auch maligne l'umoren der Thiere nicht ohne weiteres mit denen des Menschen zu identificiren sind, so bieten sie doch vom rein experimentell-pathologischen Standpunkte aus ein gewisses Interesse.

Da sich unsere eigenen Versuche an die von Braunstein anlehnen, wollen wir zunächst eine kurze Zusammenstellung seiner Resultate geben.

Um den Zeitpunkt einer eventuellen „Antikörperbildung" und die Wirkung derselben auf Mäusecarcinom zu studiren, entnahm er Carcinommäusen die Milz steril, verrieb dieselbe frisch in physiologischer Kochsalzlösung und injicirte diesen Milzbrei 3-4 mal täglich anderen Tumorthieren subcutan oder intraperitoneal. Er entnahm die Milz:

1. Thieren, die $4-5$ Tage vorher geimpft worden waren, ebe es noch zur Tumorbildung gekommen war;

2. Thieren mit bereits gut entwickelten Tumoren.

ad 1. In 5 Versuchsreihen starben von 30 Mäusen 10 Thiere während der Impfung. Bei 5 Mäusen konnte eine Rückbildung der etwa haselnussgrossen Tumoren beobachtet werden.

ad 2. Bei der 2. Versuchsreihe, wo er Milzen von Thieren mit gut entwickelten Tumoren verwendete, gingen von 15 Mäusen 6 Stück bei der Injection zu Grunde. Von den 11 überlebenden Thieren bildeten sich in 5 Fällen die Tumoren zurück, Die Tumoren hatten anfangs Erbsen- bis Haselnussgrösse. 
In einer 3. Versuchsreihe entnahm er die Milz Thieren, die 4-6 Tage vorher 2-3 mal mit Tumorbrei (Carcinom resp. Sarkom) intraperitoneal injicirt worden waren. Von 45 Krebsmäuson starben 3 während der Injection. In 6 Fällen bildeten sich baselnussgrosse Tumoren zurück, bei dreien trat Wachsthumsstillstand ein.

Bei 7 in gleicher Weise behandelten Sarkomratten gingen bei 6 die etwa pflaumengrossen Tumoren zurück.

Auf Grund seiner Versuche behauptet Braunstein:

1. Der Milz kommt eine hohe immmunisatorische Kraft gegenüber malignen Tumoren zu.

2. Splenektomirte Thiere gehen nach Behandlung mit artfremdem Krebsgewebe zu Grunde.

$\mathrm{Zu}$ ähnlichen Resultaten gelangten Rohdenburg und Johnston. Nach Injection von Extracten aus Thymus, Pankreas, Milz, Hypophyse und Hoden konnten dieselben eine Erhöhung der Immunität gegen Carcinom censtatiren. Injection derartiger Extracte hatte eine Verkleinerung resp. eine Rückbildung von Tumoren zur Folge. Durch Exstirpation von Thyreoidea, Thymus und Testis konnten diese Autoren dagegen eine Verminderung der Empfänglichkeit gegen Carcinom erzielen.

Was unsere eigenen Versuche anbelangt, so lag uns zunächst daran, festzustellen, ob durch Milzexstirpation eine Beeinflussung des Tumorwachsthums möglich sei. Besonderes Gewicht legten wir darauf, die Splenektomie stets längere Zeit vor den Tumorimpfungen (3-4 Wochen vorher) zu machen, um den Einfluss, den der operative Eingriff an und für sich hat und die dadurch bewirkte Herabsetzung der Resistenz des Organismus auszuschliessen. In einer grösseren Anzahl von Versuchen konnte einwandfrei festgestelltwerden, dass bei splenektomirten Ratten ein bedeutend rascheres Wachsthum von Sarkom gegenüber den Controlthieren eintrat.

Von den zahlreichen Versuchen, die wir zu diesem Zwecke machten, seien nur einige genauer erwähnt, um eine Vorstellung von den Wachsthums- und Grössenunterschieden der Tumoren bei Normalratten und splenektomirten Thieren zu geben.

Wir verwendeten zu unseren Experimenten ausschliesslich Ratten, die wir mit Sarkom (kleinzelliges Spindelzellensarkom) impften. Es wurden subcutane Stückimpfungen vorgenommen.

1. 6. Ratten, denen die Milz 3 Wochen vorher exstirpirt worden war, wurden mit Sarkomstückchen subcutan geimpft. Als Controlthiere dienten 6 gleichzeitig geimpfte Normalratten. Bereits 12 Tage nach der. Impfung machte sich ein Grössenunterschied bemerkbar. Während die Tumoren der splenektomirten Thiere über Walnussgrösse erreichten, waren die der Controlthiere im Maximum so gross wie eine Haselnuss. Nach 20 Tagen. wurden genauere Messungen vorgenommen. In folgender Tabelle sind die Grössenverhältnisse durch das Verhältniss des Längendurchmessers zum Querdurchmesser gegeben:

Splenektomirte Thiere: $4^{1} / 2: 4,4: 3,4: 3^{1 / 2}, 4: 3^{1} / 2,3: 2,4: 3 \mathrm{~cm}$
Normalthiere: $2: 1,2^{1 / 2}: 1^{1 / 2}, 2^{1} / 2: 2,1^{1 / 2}: 1,1^{1} / 2:{ }^{1 / 2}, 1: 1 / 2 \mathrm{~cm}$.

Mit dem weiteren Wachsthum der Tumoren traten die Grössenunterschiede noch deuthicher hervor, indem die Tumoren der splenektomirten 
Thiere viel rascher wuchsen und auch meistens erheblich grösser wurden als die der Controlthiere. Im Durchschnitt gingen die Thiere ohne Milz nach etwa 4-5 Wochen unter steter Abmagerung zu Grunde, während der Tumor wiederholt nennenswerthe Grösse erreichte (Kindsfaustgrösse und darüber). Die nachstehenden Abbildungen geben eine Vorstellung von der Grössendifferenz der Tumoren einer splenektomirten und zweier Normalratten nach 20 Tagen.

Es war nun von Interesse zu sehen, wie sich Ratten, denen die Milz exstirpirt worden war, gegenüber artfremden malignen Tumoren (Menschencarcinom und Sarkom, ferner Mäusecarcinom) im Vergleich zu den Controlthieren verhalten würden. Wir impften sowohl splenektomirte Ratten wie Normalthiere in einigen Versuchsreihen mit frisch operativ gewonnenem Mammacarcinom, Flexurcarcinom, ferner einem Sarkom des Schädels, einem Nierensarkom und schliesslich mit Mäusecarcinom. Einen sehr deutlichen Unterschied in ihrem Verhalten konnten wir in dem Falle constatiren, wo wir ein vom Menschen gewonnenes Nierensarkom zur

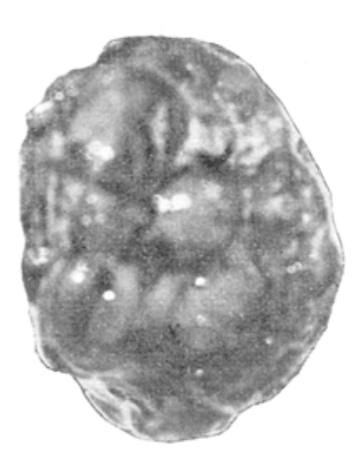

Tumor der splenektomirten Ratte.

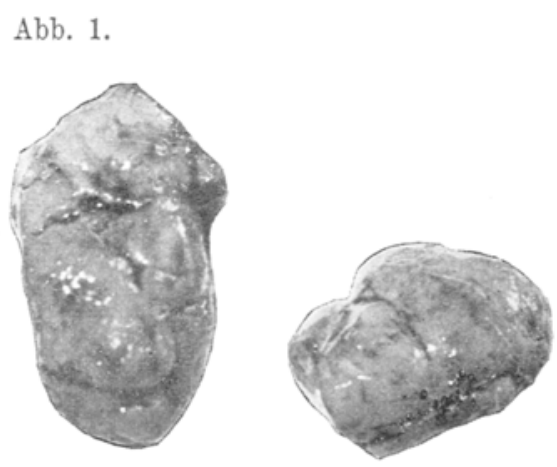

Tumoren von Normalratten.

Impfung verwendeten. Während die 6 splenektomirten Thiere innerhalb von 3 Tagen sämmtlich zu Grunde gingen, blieben alle 6 Controlthiere am Leben. Die Obduction ergab bei allen verstorbenen Thieren multiple Abscesse in den Lungen, ferner blutigen Urin in der Blase. Der Tumor war bei keiner Ratte angegangen. Bei den Controlthieren wurde das subcutan implantirte Tumorstückchen bei 5 Ratten glatt resorbirt. Bei einem Thiere bildete sich ein etwa haselnussgrosser Tumor, der aber in einigen Wochen vollständig verflüssigt wurde. Ob der Exitus der splenektomirten Ratten durch Wirkung des Nierensarkoms oder durch bakterielle Infection hervorgerufen wurde, konnten wir nicht mit Sicherheit entscheiden, jedenfalls zeigten aber die milzlosen Thiere eine bedeutende Herabsetzung der Resistenzfähigkeit des Organismus. Artfremde Tumoren gingen in keinem Falle, weder bei splenektomirten nochbei Normalratten an. Die Tumorstückchen waren entweder resorbirt oder verfüssigt.

In einer zweiten Versuchsreihe beschäftigten wir uns mit dem Einfluss von Milzbrei auf Sarkome.

Ehe wir näher auf die Versuchsresultate eingehen, sei erwähnt, dass wir besonderes Gewicht darauf legten, die Milz vollständig steril zu ent- 
nehmen. Dieselbe wurde dann mit etwas physiologischer Kochsalzlösung verrieben und zu subcutaner Injection sofort nach der Operation verwendet. $\mathrm{Zu}$ einer Injection wurde stets eine ganze Milz genommen. Um eine eventuelle specifische Wirkung des Milzbreies auf die Tumoren in exacter Weise beobachten zu können, muss man einige wichtige Punkte im Auge behalten.

Vor Allem darf der Milzbrei nicht in den Tumor selbst injicirt werden, da ja bekanntlich an und für sich indifferente Substanzen, wie z. B. physiologische Kochsalzlösung oder Methylenblau, bei localer Injection Nekrose und Rückbildung des Tumors erzeugen können. Von einer specifischen Heilwirkung kann dabei natürlich nicht die Rede sein.

Da mit dem Milzbrei auch Blut mit injicirt wird, in welchem ebenfalls leicht specifische Substanzen enthalten sein könnten, so hielten wir es zur Klärung dieser Frage für wichtig, eine Reihe von Tumorthieren nur mit Blut anderer Sarkom- und Normalratten zu injiciren.

\section{A. Injection von Milzbrei.}

Tumorratten, die 14 Tage vorher mit Sarkom geimpft worden waren und die etwa walnussgrosse Tumoren zeigten, wurde die Milz exstirpirt, in der oben erwähnten Weise verrieben und anderen Tumorthieren subcutan injicirt. $\mathrm{Zu}$ diesem Versuche wurden 4 Ratten verwendet, die annähernd gleich grosse Tumoren (walnussgross) besassen. Nach 7 Tagen zeigte sich bereits ein Grössenunterschied.

$$
\begin{array}{llll}
\text { Mit Milzbrei behandelte Thiere } & 6^{1} / 2: 6 & 9: 6 \mathrm{~cm} \\
\hline \text { Controlthiere . . . . . . } & 1^{1 / 2}: 1 & 5: 4 \mathrm{~cm}
\end{array}
$$

Es wurde nun eine zweite Injection mit Milzbrei vorgenommen.

Nach 18 Tagen war der Tumor der einen mit Milzbrei behandelten Ratte vollständig resorbirt, der der zweiten blieb auf Walnussgrösse stehen, während die Tumoren der Controlthiere sichtlich weiter wuchsen. Drei Wochen nach der ersten Injection gingen die beiden Controlthiere zu Grunde, worauf die beiden noch lebenden Ratten getödtet und die Tumoren photographirt wurden. Umstehende Abbildungen der, Ratten und der Tumoren geben ein deutliches Bild der Grössenunterschiede. Die nebeneinander aufgenommenen Ratten hatten vor Beginn des Versuches fast gleich grosse Tumoren.

Ein ähnliches Resultat ergab eine zweite Versuchsreihe.

Von zwei Ratten, die 3 Wochen vorher mit Sarkom geimpft worden waren (Tumorgrösse $4^{1 / 2}: 4 \mathrm{~cm}$ resp. $4^{1 / 2}: 5 \mathrm{~cm}$ ) wurde der einen die $\mathrm{Milz}$ einer gleichzeitig geimpften Ratte mit ungefähr gleich grossem Tumor subcutan injicirt, während die zweite als Controlthier diente.

18 Tage nachher ging das Controlthier zu Grunde. Der Tumor hatte etwa die Grösse einer Wallnuss und war etwas exulcerirt. Die mit Milzbrei behandelte Ratte blieb am Leben, der Tumor war vollständig geschwunden.

3. Versuch: Drei Ratten, denen die Milz 10 Wochen vorher exstirpirt worden war, wurden mit Sarkom geimpft und gleichzeitig drei 
Normalratten, denen aber Milzbrei von Sarkomratten (Tumor 14 Tage alt) subcutan injicirt wurde. Dazu 3 Controlthiere.

8 'Tage nach der Impfung hatten die splenektomirten 'Thiere über haselnussgrosse Tumoren. $V_{0}$ den mit Milzbrei behandelten Ratten war eine eingegangen, die beiden überlebenden wiesen keinen Tumor auf. Die Controlthiere hatten etwa bohnengrosse Sarkome.

Fassen wir das Ergebnis dieses Versuches zusammen, so zeigt sich, dass die Tumoren der splenektomirten Thiere am

Abb. 2.

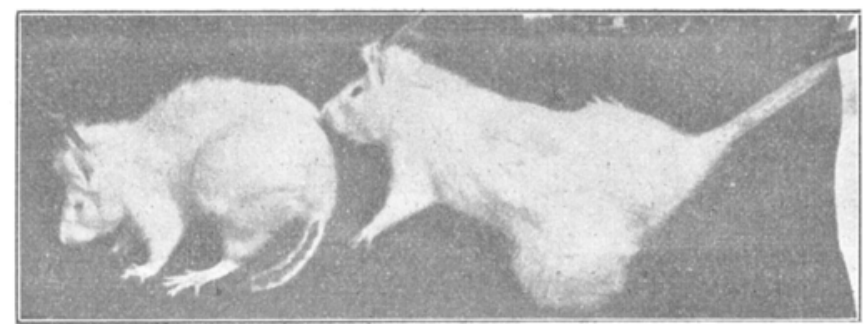

a

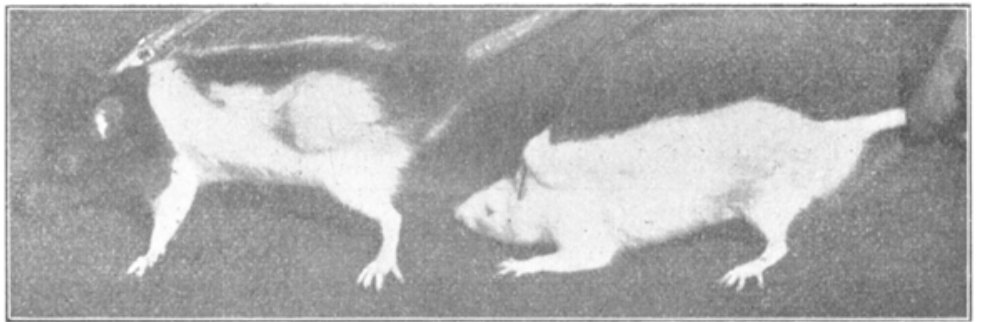

$a^{\prime}$
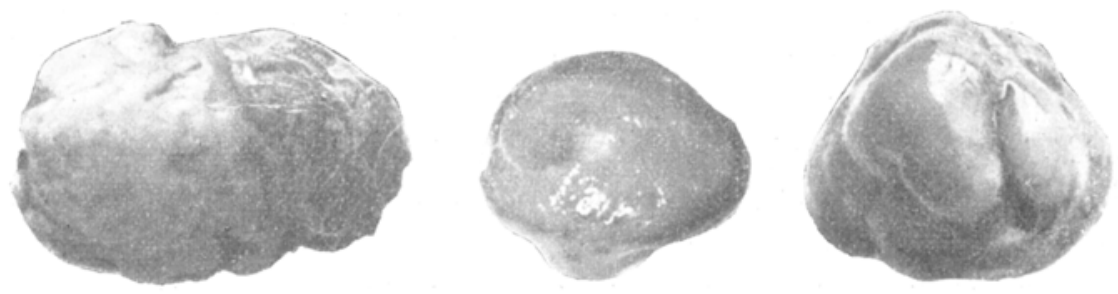

a

$a^{\prime}$

$\left.a^{\prime}\right\}$ Controlthiere und deren Tumoren. $\left.\begin{array}{l}b^{\prime} \\ b^{\prime}\end{array}\right\}$ Mit Milzbrei behandelte Thiere.

Der Tumor bei $b^{\prime}$ vollständig geschwunden.

raschesten wuchsen. Bei den gleichzeitig mit der Impfung mit Milzbrei behandelten Ratten gingen die Tumoren überhaupt nicht an.

4. Versuch: Eine Sarkomratte (Tumor wallnussgross) erhielt eine subcutane Injection von Milzbrei einer anderen Tumorratte (14 Tage vorher geimpft). Controlthier. 
Nach 12 Tagen konnte bereits eine deutliche Rückbildung des Tumors der mit Milz behandelten Ratte constatirt werden.

22 Tage nach Beginn des Versuches starb die Controlratte, deren Tumor apfelgross war. Der Tumor der mit Milzbrei geimpften Ratte war vollständig geschwunden.

5. Versuch: 2 Tumorratten (Tumor 14 Tage alt) wurde Milzbrei injicirt, der von Ratten stammte, die 4 Tage vorher mit Sarkom geimpft worden waren. Controlthiere. 14 Tage nachher noch keine wesentlichen Grössenunterschiede zu constatiren.

6. Versuch: Einer Ratte, die einen etwa apfelgrossen Tumor hatte, wurde Sarkommilzbrei injicirt. Innerhalb von 3 Wochen wurden 4 Impfungen vorgenommen. Die Milz wurde in diesem Versuche wieder von Ratten genommen, die bereits gut entwickelte Tumoren besassen. Eine Rückbildung oder auch nur Stillstand im Wachstum des Sarkoms konnte nicht erreicht werden. Die Ratte starb schliesslich 8 Wochen nach der Impfung unter starker Abmagerung. Ihr Tumor hatte eine Grösse von $10: 71 / 2 \mathrm{~cm}$ erreicht. Auffallend war, dass die Ratte relativ lange am Leben blieb.

Aus den beiden letzten Versuchen ersieht man, dass nicht immer eine, Beeinflussung der Tumoren durch Milzbrei zu erzielen ist. Die Milzexstirpation in Versuch $V$ war wahrscheinlich zu kurze Zeit nach der Impfung vorgenommen worden. In Versuch VI dürfte der Tumor der Ratte bereits zu weit vorgeschritten gewesen sein. Durch Milz von Normalratten konnte eine Wirkung auf Tumoren nicht erzielt werden.

Wir beschäftigten uns schliesslich mit der Wirkung des Blutes von Normal- und Sarkomratten auf Tumoren.

\section{B. Blutversuche.}

Das nicht defibrinirte Blut von Sarkomratten wurde sofort nach Entnahme mittelst einer Pravazspritze subcutan injicirt. Eine Wirkung auf den Tumor konnten wir nicht erzielen. Dasselbe gilt auch rom Blut von Normalratten.

Eine interessante Beobachtung sei jedoch an dieser Stelle kurz mitgetheilt. Bei einer grösseren Anzahl von Thieren versuchten wir, nicht defibrinirtes Blut von Sarkomratten in den Tumor selbst einzuspritzen. Innerhalb von $2-3$ Secunden nach der Injection $\nabla$ on 0,5 bis $1 \frac{1}{2}$ cem Blut gingen die Thiere stets unter Zeichen von Dyspnoe zu Grunde, während das Herz noch einige Secunden länger schlug. Es war naheliegend, daran zu denken, dass in dem Blute der Sarkomratten eine besonders giftige Substanz enthalten sein könnte, die vom Tumor aus sehr rasch resorbirt wurde und auf diese Weise den plötzlichen Tod des Thieres herbeiführt. Da jedoch auch Blut von Normalratten dieselbe Wirkung hatte, da ferner defibrinirtes Blut bei Injection in den Tumor von den Ratten sehr gut vertragen wurde, müssen doch andere Factoren dabei maassgebend sein. Nach unserer Ansicht handelt es sich hier um Embolien. Bei der reichen Vascularisation des Tumors ist eine 
302 E. G. Oser a. Egon Ewald Pribram, Ueber die Bedeutung der Milz etc.

Verletzung eines Gefässes kaum zu vermeiden. Ein kleines Coagulum geronnenen Blutes könnte dann leicht zu einer Embolie führen.

Wir behalten uns vor, noch genauere Untersuchungen darüber anzustellen und demnächst mitzuteilen und haben diese Beobachtung hauptsächlich deshalb hier erwähnt, um in Anbetracht der grossen Gefahr, die eine Injection in den Tumor selbst bietet, auch bei therapeutischen Versuchen am Menschen zur Vorsicht zu mahnen und vor Einspritzungen in maligne Geschwülste zu warnen. Da durch subcutane Injection von Blut eine Beeinflussung des Tumorwachstums nicht zu erzielen war, hat es den Anschein, dass in der Milz der Sarkomratten Substanzen vorhanden sind, die einen Tumor zur Rückbildung oder zu Wachsthumsstillstand bringen, während das gleichzeitig entnommene Blut dieselben nicht enthält.

\section{Résumé.}

1. Der Milz kommt eine grosse Bedeutung in dem an malignem Tumor erkrankten Organismus zu.

2. Splenektomirte Ratten zeigen ein rascheres Tumorwachsthum.

3. Durch Injection von Milzbrei kann bei Sarkomratten eine Rückbildung oder Wachsthumsstillstand des Tumors bewirkt werden.

4. Es scheint dies durch Substanzen (vielleicht nach Art der Antikörper) herbeigeführt zu werden, die im gleichzeitig entnommenen Blute nicht vorhanden sind.

5. Injectionen in maligne Tumoren selbst sind zu vermeiden.

\section{Litteratur.}

Oestreich, Berliner klin. Wochenschr. 1910. No. 37.

Braunstein, Berliner klin. Wochenschr. 1911. No. 45. 6. September. Rohdenburg u. Johnston, Arch. of. intern. med. 1911. No. 4. 